\title{
Respiratory System Findings Sponsor Defined Identifier
}

National Cancer Institute

\section{Source}

National Cancer Institute. Respiratory System Findings Sponsor Defined Identifier. NCI

Thesaurus. Code C124005.

One or more sponsor defined characters used to identify, name, or characterize the respiratory system findings. 